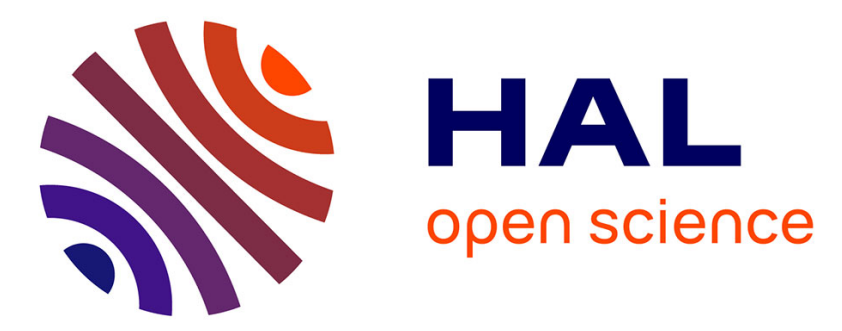

\title{
A polyhedral approach to locating-dominating sets in graphs
}

Gabriela R Argiroffo, Silvia Bianchi, Annegret K Wagler

\section{To cite this version:}

Gabriela R Argiroffo, Silvia Bianchi, Annegret K Wagler. A polyhedral approach to locatingdominating sets in graphs. Electronic Notes in Discrete Mathematics, 2015, 50, pp.89-94. hal02045771

\section{HAL Id: hal-02045771 \\ https://hal.science/hal-02045771}

Submitted on 22 Feb 2019

HAL is a multi-disciplinary open access archive for the deposit and dissemination of scientific research documents, whether they are published or not. The documents may come from teaching and research institutions in France or abroad, or from public or private research centers.
L'archive ouverte pluridisciplinaire HAL, est destinée au dépôt et à la diffusion de documents scientifiques de niveau recherche, publiés ou non, émanant des établissements d'enseignement et de recherche français ou étrangers, des laboratoires publics ou privés. 


\title{
A polyhedral approach to locating-dominating sets in graphs
}

\author{
Gabriela R. Argiroffo ${ }^{1}$, Silvia M. Bianchi ${ }^{2}$ \\ Universidad Nacional de Rosario (Dept. de Matemática), Rosario, Argentina \\ Annegret K. Wagler ${ }^{3}$ \\ University Blaise Pascal (LIMOS, UMR 6158 CNRS), Clermont-Ferrand, France
}

\begin{abstract}
The locating-dominating set problem is a special domination problem, challenging both from a theoretical and a computational point of view. Hence, a typical line of attack is to determine minimum locating-dominating sets of special graphs or to provide bounds for their size. Here, we study the locating-dominating set problem from a polyhedral point of view and demonstrate how the associated polyhedra can be entirely described for some basic families of graphs. The latter enables us to determine minimum weight locating-dominating sets in the studied graph classes for arbitrary integral node weights. We discuss further lines of research in order to apply similar techniques for other graph classes, to obtain the exact values or strong lower bounds for the size of minimum locating-dominating sets, stemming from linear relaxations of the polyhedra, enhanced by suitable cutting planes.
\end{abstract}

Keywords: set covering, locating-dominating set, facet-descriptions of polyhedra

$\overline{1 \text { Email: }}$ garua@fceia.unr.edu.ar

2 Email: sbianchi@fceia.unr.edu.ar

3 Email: wagler@isima.fr 


\section{Introduction}

Many practical problems can be formulated to find certain combinatorial substructures in related graphs. Some safeguard applications of facilities as, e.g., placing detectors for fault detection in networks or motion/fire detection in buildings, can be modeled by so-called locating-dominating sets in graphs.

In a graph $G=(V, E)$, the open neighborhood $N(i)$ of $i \in V$ is the set of all nodes adjacent to $i$ and $N[i]=\{i\} \cup N(i)$ is the closed neighborhood of $i$.

Slater $[7,8]$ defined a locating-dominating set in a connected graph $G$ to be a node subset $C$ such that $N(i) \cap C$ and $N(j) \cap C$ are non-empty and different sets for all $i \neq j \in V-C$. By definition, $V$ is trivially a locating-dominating set in $G=(V, E)$. The locating-dominating number $\gamma^{L D}(G)$ of a graph $G$ is the minimum cardinality of a locating-dominating set of $G$.

Determining $\gamma^{L D}(G)$ is in general NP-complete [3]. From a graph theoretical point of view, the problem was studied in $[4,5,7,8]$ among others. To apply polyhedral methods, a reformulation as set covering problem is in order. For a 0/1-matrix $M$ with $n$ columns, the set covering polyhedron is $Q^{*}(M)=\operatorname{conv}\left\{x \in \mathbb{Z}_{+}^{n}: M x \geq 1\right\}$ and $Q(M)=\left\{x \in \mathbb{R}_{+}^{n}: M x \geq 1\right\}$ is its linear relaxation. A cover of $M$ is a $0 / 1$-vector $x$ such that $M x \geq \mathbf{1}$, and the covering number $\tau(M)$ equals $\min \mathbf{1}^{T} x, x \in Q^{*}(M)$. We obtain such a constraint system $M x \geq \mathbf{1}$ for the locating dominating set problem as follows:

Theorem 1.1 $C$ is a locating-dominating set of $G=(V, E)$ if and only if its characteristic vector $x$ satisfies

(i) $x(N[i]) \geq 1$ for all $i \in V$, and

(ii) $x(N(i) \triangle N(j)) \geq 1$ for all $i, j \in V$ with $\operatorname{dist}(i, j)=1$,

(iii) $x(N[i] \triangle N[j]) \geq 1$ for all $i, j \in V$ with $\operatorname{dist}(i, j)=2$.

As the matrix encoding the constraints from Theorem 1.1 may contain rows which are equal to or dominated by other rows in it, we define the corresponding clutter matrix, the locating-dominating set clutter $C_{L D}(G)$ of $G$, obtained by removing repeated or dominated rows. We define the locating-dominating set polyhedron as $P_{L D}(G)=Q^{*}\left(C_{L D}(G)\right)=\operatorname{conv}\left\{x \in \mathbb{Z}_{+}^{|V|}: C_{L D}(G) x \geq \mathbf{1}\right\}$, and its linear relaxation as $Q_{L D}(G)=Q\left(C_{L D}(G)\right)$. Determining $\gamma^{L D}(G)$ can be done by solving the set covering problem $\gamma^{L D}(G)=\tau\left(C_{L D}(G)\right)=$ $\min \left\{\mathbf{1}^{T} x: x \in P_{L D}(G)\right\}$.

Due to the hardness of the problem, we cannot expect to find a complete facet description of $P_{L D}(G)$ for all graphs $G$ (which would allow us to solve the weighted problem by linear programming techniques). 
So we propose to determine the locating-dominating set clutter $C_{L D}(G)$ for special graphs and then to study which constraints have to be added to $Q_{L D}(G)$ in order to strengthen the linear relaxation. That way, we provide the complete description of $P_{L D}(G)$ for complete $p$-partite graphs (Section 2) and thin suns (Section 4 ), and formulate an according conjecture for paths (Section 3). Finally, we discuss some consequences and future lines of research.

\section{Complete multipartite graphs}

Consider complete $p$-partite graphs $K_{n_{1}, \ldots, n_{p}}=\left(V_{1}, \ldots, V_{p}, E\right)$ where each $V_{i}$ induces a stable set, all edges between any $V_{i}$ and $V_{j}$ are present, $\left|V_{i}\right|=n_{i}$ for $i=1, \ldots, p$ and $|V|=n$. We establish a connection between $C_{L D}\left(K_{n_{1}, \ldots, n_{p}}\right)$ and matrices $R_{n}^{2}$ having as rows all the possible 0/1-vectors with two 1entries, called complete 2-roses of order $n$ by Sassano [6]. Using the complete facet description of $Q^{*}\left(R_{n}^{2}\right)$ from [1], we can provide such a description for $P_{L D}\left(K_{n_{1}, \ldots, n_{p}}\right)$ which allows us to determine $\gamma^{L D}\left(K_{n_{1}, \ldots, n_{p}}\right)$ directly (and the weighted version by linear programming techniques).

We start with the two special cases of stars $K_{1, n_{2}}$ (i.e., $n_{1}=1, p=2$ ) and cliques $K_{p}$ (i.e., $n_{1}=\cdots=n_{p}=1$ ).

Lemma 2.1 If $G=(V, E)$ is a star $K_{1, n-1}$ or a clique $K_{n}$, we have

(i) $C_{L D}(G)=R_{n}^{2}$,

(ii) $P_{L D}(G)$ is described by the inequalities $x\left(V^{\prime}\right) \geq\left|V^{\prime}\right|-1$ for all nonempty subsets $V^{\prime} \subseteq V$, and, thus, $\gamma^{L D}(G)=n-1$.

The above inequalities yield for $\left|V^{\prime}\right|=1$ and $\left|V^{\prime}\right|=2$ the inequalities describing $Q_{L D}(G)$. On the other hand, $V^{\prime}=V$ yields the full rank facet $x(V) \geq|V|-1$ which immediately implies $\gamma^{L D}(G)=|V|-1$.

Lemma 2.2 For a complete p-partite graph $K_{n_{1}, n_{2}, \ldots, n_{p}}$ with $n_{1}=1, n_{i} \geq 2$ for $i=2, \ldots, p$, and $p>2$, we have

$$
C_{L D}\left(K_{1, n_{2}, \ldots, n_{p}}\right)=\left(\begin{array}{cccc}
0 & R_{n_{2}}^{2} & \ldots & 0 \\
\vdots & & \ddots & \vdots \\
0 & \ldots & 0 & R_{n_{p}}^{2}
\end{array}\right)
$$

$P_{L D}\left(K_{1, n_{2}, \ldots, n_{p}}\right)$ is described by the inequalities $x\left(V^{\prime}\right) \geq\left|V^{\prime}\right|-1$ for all nonempty subsets $V^{\prime} \subseteq V_{i}$ for $i=2, \ldots, p$, and $\gamma^{L D}\left(K_{1, n_{2}, \ldots, n_{p}}\right)=n-p$. 
Lemma 2.3 For a complete p-partite graph $K_{n_{1}, n_{2}, \ldots, n_{p}}$ with $n_{1}=\cdots=n_{r}=$ $1, n_{i} \geq 2$ for $i=r+1, \ldots, p$ and $1<r<p$, we have

$$
C_{L D}\left(K_{1, \ldots, 1, n_{r+1}, \ldots, n_{p}}\right)=\left(\begin{array}{cccc}
R_{r}^{2} & 0 & \ldots & 0 \\
0 & R_{n_{r+1}}^{2} & \ldots & 0 \\
\vdots & & \ddots & \vdots \\
0 & \ldots & 0 & R_{n_{p}}^{2}
\end{array}\right)
$$

$P_{L D}\left(K_{1, \ldots, 1, n_{r+1}, \ldots, n_{p}}\right)$ is given by $x\left(V^{\prime}\right) \geq\left|V^{\prime}\right|-1$ for all nonempty $V^{\prime} \subseteq V_{1} \cup$ $\cdots \cup V_{r}$ and $V^{\prime} \subseteq V_{i}$ for $i=r+1, \ldots, p$, and $\gamma^{L D}\left(K_{1, \ldots, 1, n_{r+1}, \ldots, n_{p}}\right)=n-p+r-1$.

The latter result includes complete split graphs since $K_{1, \ldots, 1, n_{p}}$ equals $G=$ $(C \cup S, E)$ where $C=V_{1} \cup \cdots \cup V_{p-1}$ induces a clique and $S=V_{p}$ a stable set.

Lemma 2.4 For a complete p-partite graph $K_{n_{1}, n_{2}, \ldots, n_{p}}$ with $n_{i} \geq 2$ for $i=$ $1, \ldots, p$, we have

$$
C_{L D}\left(K_{n_{1}, \ldots, n_{p}}\right)=\left(\begin{array}{cccc}
R_{n_{1}}^{2} & 0 & \ldots & 0 \\
0 & R_{n_{2}}^{2} & \ldots & 0 \\
\vdots & & \ddots & \vdots \\
0 & \ldots & 0 & R_{n_{p}}^{2}
\end{array}\right)
$$

$P_{L D}\left(K_{n_{1}, \ldots, n_{p}}\right)$ is described by the inequalities $x\left(V^{\prime}\right) \geq\left|V^{\prime}\right|-1$ for all nonempty subsets $V^{\prime} \subseteq V_{i}$ for $i=1, \ldots, p$, and $\gamma^{L D}\left(K_{n_{1}, \ldots, n_{p}}\right)=n-p$.

Note that the latter result includes complete bipartite graphs $K_{n_{1}, n_{2}}$.

If $G$ is a complete $p$-partite graph different from a star or a clique, $P_{L D}(G)$ does not have the full rank facet $x(V) \geq \gamma^{L D}(G)$. But combining the constraints $x\left(V^{\prime}\right) \geq\left|V^{\prime}\right|-1$ from the previous lemmas for the largest possible subsets $V^{\prime}$ yields that $x(V) \geq \gamma^{L D}(G)$ is valid for the respective values. It is easy to find a locating-dominating set of the same size, which shows that the values are indeed tight.

\section{Paths}

Consider paths $P_{n}$ with node set $\{1, \ldots, n\}$ and edge set $\{12, \ldots, n-1 n\}$. We construct $C_{L D}\left(P_{n}\right)$ and the resulting linear relaxation $Q_{L D}\left(P_{n}\right)$. 
Lemma 3.1 For a path $P_{n}$ with $n \geq 5, Q_{L D}\left(P_{n}\right)$ is given by the

- trivial inequalities $x_{i} \geq 0$ for all $i \in V\left(P_{n}\right)$,

- closed neighborhood inequalities $x_{1}+x_{2} \geq 1, x_{i}+x_{i+1}+x_{i+2} \geq 1$ for $3 \leq i \leq n-3$, and $x_{n-1}+x_{n} \geq 1$,

- closed symmetric difference inequalities $x_{1}+x_{3}+x_{4} \geq 1, x_{n-3}+x_{n-2}+x_{n} \geq 1$ and $x_{i-1}+x_{i}+x_{i+2}+x_{i+3} \geq 1$ for all $3 \leq i \leq n-4$.

Theorem 3.2 For a path $P_{n}$ with $n \geq 5, P_{L D}\left(P_{n}\right)$ has as facets

- $x_{1}+x_{2}+x_{3}+x_{4} \geq 2, x_{n-3}+x_{n-2}+x_{n-1}+x_{n} \geq 2$,

- $x_{i}+x_{i+1}+x_{i+2}+x_{i+3}+x_{i+4} \geq 2$ for $i=2, \ldots, n-5$.

It is known that $\gamma^{L D}\left(P_{n}\right)=\left\lceil\frac{2 n}{5}\right\rceil$ [8]. It is easy to see that combining the inequalities in Theorem 3.2 for every $i=1, \ldots, n$ yields that $x(V) \geq \frac{2 n}{5}$ is valid for $P_{L D}\left(P_{n}\right)$. If we round up the right hand side, we obtain $\gamma^{L D}\left(P_{n}\right)$.

Conjecture 3.3 The complete description of the polyhedron $P_{L D}\left(P_{n}\right)$ is obtained by adding all facets in Theorem 3.2 to the inequalities in $Q_{L D}\left(P_{n}\right)$.

This conjecture has been verified in some experiments for $n \leq 20$.

\section{Thin suns}

A sun is a graph $G=(C \cup S, E)$ whose node set can be partitioned into $S$ and $C$, where $S=\left\{s_{1}, \ldots, s_{n}\right\}$ is a stable set and $C=\left\{c_{1}, \ldots, c_{n}\right\}$ a (not necessarily chordless) cycle. A thin sun $T_{n}=(C \cup S, E)$ is a sun where $s_{i}$ is adjacent to $c_{j}$ if and only if $i=j$.

Lemma 4.1 For a thin sun $T_{n}$, we have $C_{L D}\left(T_{n}\right)=(I, I)$ and $P_{L D}\left(T_{n}\right)$ coincides with its linear relaxation $Q\left(C_{L D}\left(T_{n}\right)\right)$.

Adding up all constraints from the system $C_{L D}\left(T_{n}\right) x \geq \mathbf{1}$ yields the valid full rank inequality $x(C \cup S) \geq n$. On the other hand, both $C$ and $S$ are clearly locating-dominating sets of $T_{n}$. This immediately implies:

Corollary 4.2 For $T_{n}$ with $n \geq 4, \gamma^{L D}\left(T_{n}\right)=n$ holds.

These results are independent from the subgraph that $C$ induces in $T_{n}$ and apply particularly to thin suns where $C$ induces a hole, called sunlets.

\section{Conclusions}

In this contribution, we studied the locating-dominating set problem from a polyhedral point of view. For that, we firstly reformulated the problem in 
terms of set covering. For all considered graph families, we determined the resulting locating-dominating set clutters and provided the complete description of $P_{L D}(G)$ for all cases of complete $p$-partite graphs and thin suns, and formulate an according conjecture for paths.

On the one hand, these complete descriptions allow us to compute the exact value of the locating-dominating number (generalizing some results from [8]) and even extend to the weighted version. On the other hand, they provide us with information about the relation between $Q^{*}\left(C_{L D}(G)\right)$ and its linear relaxation $Q\left(C_{L D}(G)\right)$ : we identified a case where $C_{L D}(G)$ is an ideal matrix by $Q^{*}\left(C_{L D}(G)\right)=Q\left(C_{L D}(G)\right)$.

A further goal is to identify the full rank constraint $x(V) \geq \gamma^{L D}(G)$ for $P_{L D}(G)$ (e.g., by means of the Chvátal-Gomory procedure) in order to obtain the exact value of the locating-dominating number for other graphs $G$ where $C_{L D}(G)$ is not ideal. Note that this is a general approach based on polyhedral arguments which can be applied to the locating-dominating set polyhedra of all graphs, even if the system of facet-defining inequalities is very complex.

\section{References}

[1] G. Argiroffo, M. Carr, On the set covering polyhedron of q-roses, VI ALIO/EURO Workshop on Applied Combinatorial Optimization 2008.

[2] N. Bertrand, I. Charon, O. Hudry and A. Lobstein, Identifying and locatingdominating codes on chains and cycles, Eur. J. Combin., 25 (2004) 969-987.

[3] I. Charon, O. Hudry and A. Lobstein, Minimizing the size of an identifying or locating- dominating code in a graph is NP-hard, Theor. Comput. Sci., 290 (2003) 2109-2120.

[4] C.J. Colbourn, P.J. Slater, L.K. Stewart, Locating-dominating sets in seriesparallel networks, Congr. Numer. 56 (1987) 135-162.

[5] T.W. Haynes, M.A. Henning, J. Howard, Locating and total dominating sets in trees, Discrete Applied Mathematics 154 (2006) 1293-1300.

[6] A. Sassano, On the facial structure of the set covering polytope, Mathematical Programming 44 (1989) 181-202.

[7] P.J. Slater Dominating and location in acyclic graphs, Networks 17 (1987) 55-64.

[8] P.J. Slater, Dominating and reference sets in a graph, J. Math. Phys. Sci. 22 (1988) 445-455. 